\title{
An online e-Learning authoring tool to create interactive multi-device learning objects using e-Infrastructure resources
}

\author{
Aldo Gordillo, Enrique Barra, Daniel Gallego and Juan Quemada \\ Escuela Técnica Superior de Ingenieros de Telecomunicación \\ Universidad Politécnica de Madrid \\ Avenida Complutense 30, 28040, Madrid, Spain \\ \{agordillo, ebarra, dgallego, jquemada\}@dit.upm.es
}

\begin{abstract}
Education can take advantage of e-Infrastructures to provide teachers with new opportunities to increase students' motivation and engagement while they learn. Nevertheless, teachers need to find, integrate and customize the resources provided by e-Infrastructures in an easy way. This paper presents ViSH Editor, an innovative web-based e-Learning authoring tool that aims to allow teachers to create new learning objects using e-Infrastructure resources. These new learning objects are called Virtual Excursions and are created as reusable, granular and interoperable learning objects. This way they can be reused to build new ones and they can be integrated in websites or Learning Management Systems. Details about the design, development and the tool itself are explained in this paper as well as the concept, structure and metadata of the new learning objects. Lastly, some real examples of how to enrich learning using Virtual Excursions are exposed.
\end{abstract}

Keywords - authoring tool; learning objects; e-Infrastructures

\section{INTRODUCTION}

The European Commission considers that e-Infrastructures are essential to foster scientific excellence and stimulate the development of high-quality human capital and therefore they must have a place in education and training [1]. According to [2] an e-Infrastructure consists of computational systems, data management, advanced instruments, visualization environments and people, all linked together by software and advanced networks to improve scholarly productivity and enable knowledge breakthroughs and discoveries not otherwise possible. In education, e-Infrastructures can be used to provide unprecedented access to educational resources including experts, online activities, games and virtual environments [3]. Therefore, e-Infrastructures can greatly expand learning and teaching opportunities for students and teachers [4]. For instance, they can allow interacting in real time with resources such as microscopes, virtual laboratories, webcams or tools of professional science like simulations, data sets or remote equipment. Teachers can combine virtual resources like these and traditional F2F (Face to Face) teaching in order to enrich the learning experience in an effective way taking advantage of technology without renouncing to the benefits of in-person teaching [5]. Hence, e-Infrastructures can be used in blended learning, supplementing traditional classroom activities and providing enhanced learning materials. According to [6], this is especially useful for science, technology, engineering and mathematics (STEM) disciplines as the best way to provide this kind of education is to use a blended approach.

Nevertheless, it is difficult to find accessible educational e-Infrastructure resources. Moreover, although sometimes teachers can find an appropriate one, they need to customize and link it with other educational contents (e.g. text, images or videos) in order to make it more pedagogical and to adapt it to different knowledge levels and requirements of different classes. Additionally, if teachers want to improve the quality of their own teaching materials by using e-Infrastructure resources, they should integrate both. Therefore in spite of all e-Infrastructure benefits in education, teachers usually end up using classical resources, which provide less engaging experiences, or generating their own resources from scratch.

Taking all this into account, providing e-Infrastructure resources as content units that can be individually adapted and integrated into existing teaching materials and curricula of teachers was identified as the main challenge when the GLOBAL excursion (Extended Curriculum for Science Infrastructure Online) project started. GLOBAL excursion is a European project which main aim is to enrich science teaching in European schools. Via a central web portal, called Virtual Science Hub (ViSH), we provide scientists, teachers and their pupils a package of activities, materials and tools for enabling the integration of e-Infrastructures into school curricula.

In order to allow teachers to create enhanced learning materials through integrating e-Infrastructure resources we have developed an e-Learning authoring tool called ViSH Editor. This tool will be presented in this paper. The content generated by this authoring tool is a new learning object called Virtual Excursion which will be also explained.

The rest of the paper is organized as follows. The next section reviews related work of authoring tools as well as learning objects. Section 3 introduces the GLOBAL excursion project. Section 4 shows an overview of the ViSH platform. Section 5 and 6 explain the generated learning object and the ViSH Editor authoring tool. The last section finishes with some concluding remarks together with an outlook on future work. 


\section{RELATED WORK}

Before content can be accessed, shared or reused, it must be created. Authoring tools aim to enable authors to create this content. An authoring tool can be defined as a software application that allows authors to create their own content and deliver it to the end users. As a general rule in the case of an e-Learning authoring tool, the authors are teachers, the end users are students and the content is a learning resource created following a learning object content model. An official definition of learning object (hereafter LO) can be found in the learning object metadata (LOM) standard [7], which defines a LO as "any entity, digital or non digital that may be used for learning, education or training". However, this is not an universal definition since LO definitions and specifications considerably vary depending on the content model [8]. The primary purpose for creating these resources as LOs is to facilitate reuse [9]. The major benefit of reusing LOs is to reduce time and to enable cost-effective development by reusing learning materials instead of repeatedly authoring them. Moreover, digital LOs may even enhance the quality in comparison with those developed from scratch [10]. The potential of a LO for reuse increases as its size or granularity decreases [11]. Thereby learning resources should be sufficiently large to be of educational value but also be small enough to be effectively reused [12]. LOs can be combined among them to build more complex ones forming a hierarchy. For instance, a slideshow can be created by linking single slides together. Furthermore, each of these slides can be created at the same time by linking other LOs such as a paragraph of text, a picture or a video. This creation process can be referred to as authoring by aggregation [11]. Also, these LOs are known as granular LOs and the different hierarchy levels are called aggregation or granularity levels. Learning object models define these levels of granularity and specify how the components can be aggregated as well as the properties of these components [13]. One very important aspect of LOs is metadata, where a description of the content is included in order to improve their reusability, discoverability and interoperability. Standards also play an essential role for an authoring tool to achieve the interoperability of the resulting LOs. For this reason, the learning resources created by most authoring tools can be exported or are built conforming to some e-Learning standard such as SCORM (Sharable Content Object Reference Model) [14] or AICC (Aviation Industry Computer-Based Training Committee). However, there also exist some e-Learning authoring tools that do not include support for e-learning standards. Since authoring tools offer a diverse variety of features, the process of selecting one to create the LOs might be hard. As [15] points out, the criteria for choosing the most suitable authoring tool should be based on the particular needs of the end users, taking into account capabilities such as automated programming, interoperability, standards, question types and media and file support. This is especially important in low-tech teaching contexts, where teachers are not computer specialists and also have poor IT (Information Technology) support. Under these conditions, it is still possible to get excellent results by using the appropriate models and authoring tools [16]. An authoring tool can provide a differentiating value not only adapting to their target users' needs but also by integrating novel learning resources such as e-Infrastructures.

Several e-Infrastructures (e.g. DEISA [17], EGI [18], OSG [19], XSEDE [20]) exist nowadays with the foremost aim of sharing computing resources and data between scientists. However, the potential impact of e-Infrastructures in education is at least as significant as that in other disciplines [4], and hence, as time goes by, more projects [21] [22] are carried out to provide more e-Infrastructures for education. Finally, bearing in mind that a LO can be formed by aggregation, these e-Infrastructure resources can be linked with other LOs through authoring tools to increase their educational value.

\section{GLOBAL EXCURSION PROJECT}

The European Commission has defined the advancement of STEM related skills as one of the priorities for the period 2014-2020 [23]. Furthermore, it has shown in several reports its concern about the way science teaching is currently being performed [1]. Bearing all this in mind, and considering that e-Infrastructures are recognized by the European Commission as a key to a knowledge-based economy and social cohesion that need to have a place in education, the GLOBAL excursion project (http://www.globalexcursion-project.eu) was proposed and approved.

Together with end users, GLOBAL excursion develops a common understanding, teaching use cases, as well as pedagogical and technical artifacts. The aim of this project is to provide students and their educators (teachers, parents, etc.) across Europe with a range of e-Infrastructures and access to expert knowledge on its usage for a joyful exploration of e-Science. Another goal of the project is to improve science curricula by enriching school's existing teaching and learning materials.

The e-Infrastructure providers participating in the project were initially three, the Institute for Biocomputation and Physics of Complex Systems (BIFI) from Spain, the Nanoscience Centre from the University of Cambridge (UCAM) from the United Kingdom and the Computer and Automation Research Institute (SZTAKI) from Hungary. Other scientific centers are expected to participate in the near future. The materials currently provided by these partners are based on the following topics: biotechnology and biology from BIFI, grid computing and volunteer computing from STZAKI and nanoscience from UCAM.

\section{VIRTUAL SCIENCE Hub}

Virtual Science Hub, or ViSH in short form, is a social collaborative e-Learning platform in which all GLOBAL excursion activities take place. It provides a social network where teachers and scientists can share their resources, know each other and collaborate. The other main aim of the ViSH platform is to create and share enhanced LOs taking advantage of e-Infrastructures for education, providing this way more engaging experiences to students. These new LOs, which we have called Virtual Excursions, are created using the ViSH Editor authoring tool. Fig. 1 represents the different actions that are carried out to create a Virtual Excursion. 


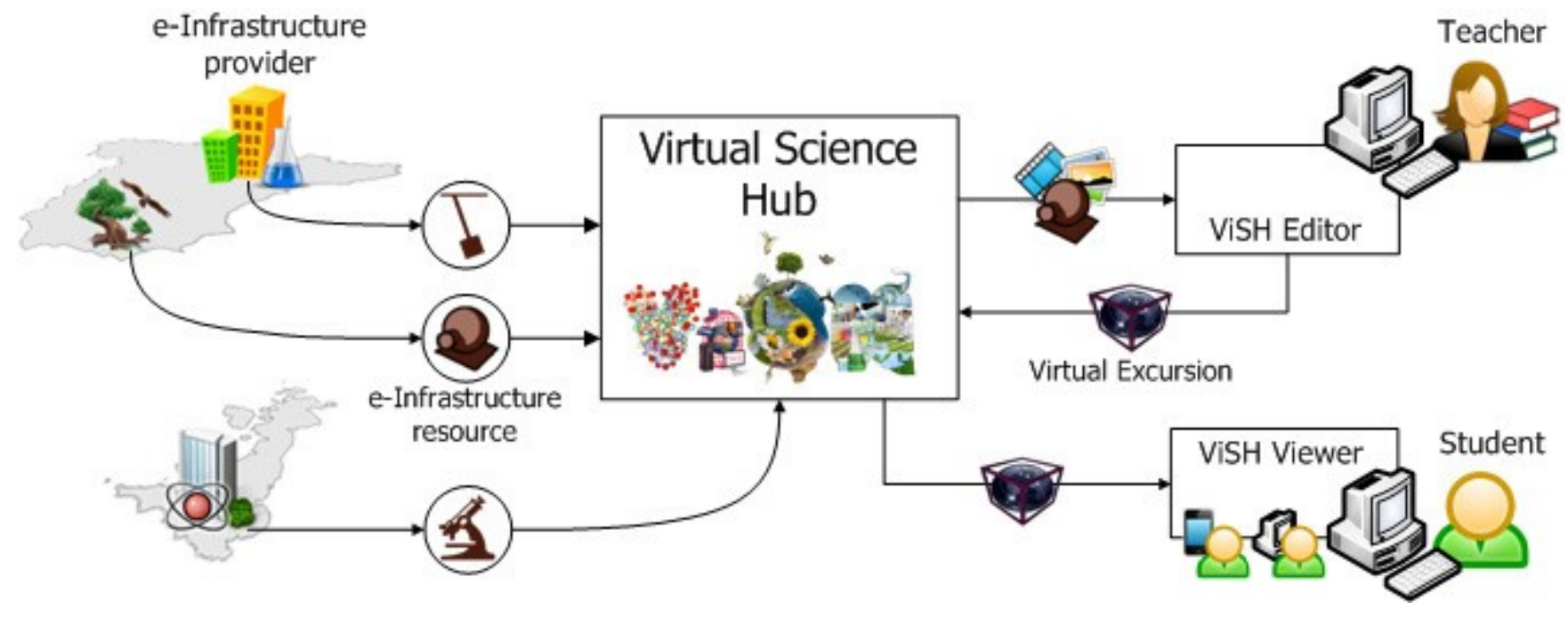

Fig. 1. Virtual Excursion generation

Besides providing the social network and the authoring tool, ViSH also provides a learning object repository (LOR) that stores both LOs and their metadata. The e-Infrastructure providers use this LOR to upload their resources. Once the e-Infrastructure resources have been stored in the LOR, they are available to be used in any Virtual Excursion. Teachers can create a Virtual Excursion online using the ViSH Editor web-based authoring tool. They can add any resource of the LOR as well as resources from external services like Flickr or YouTube to compose the excursion. When teachers finish the creation task, they can publish the excursion into the ViSH or save it as a draft for keeping it private and finishing it later. Anyway, the excursion created is stored in the ViSH LOR with the rest of the LOs. From the moment an excursion is published, it can be accessed by anyone, even without being registered in the platform. The published Virtual Excursions can also be cloned by other registered users, and of course, they can be edited or removed by their owners at any time.

To explore a Virtual Excursion users just have to load a lightweight web application called ViSH Viewer [24]. ViSH Viewer is a module of ViSH Editor used for previewing the Virtual Excursions while they are being created, but it can also work standalone. It allows users to view and interact with the Virtual Excursions (or with any other LO generated via ViSH Editor) from any device using only a web browser without any installation or previous configuration being needed. For this reason, we can also refer to the LOs generated by ViSH Editor as multi-device LOs. Using a lightweight web application is especially important for handled devices which usually have lower power processing.

ViSH has been completely designed by the project members following a participatory design process [25] whereby the user requirements were identified. It is open source and has been developed using the latest technologies on top of the social network framework Social Stream [26], which is also open source and provides common social network features such as contacts, walls, posts, comments, documents, privacy and permissions management, private and instant messaging, groups and so on.
ViSH is currently in production at http://vishub.org. It is free for end users to register, enter the community and enjoy the experience.

\section{THE VIRTUAL EXCURSIONS}

\section{A. Definition}

Conceptually, a Virtual Excursion is defined as a tour through some digital context by teachers and pupils on a given topic that is attractive and has an educational purpose [27]. A Virtual Excursion provides a new way to explore science in class allowing students and teachers to access and control experimental equipment of research laboratories at remote sites, explore natural parks, museums or any other infrastructure with educational or cultural interest which would be too expensive or non-viable to visit in person [24]. In practice, Virtual Excursions are created by teachers or scientific organizations using the ViSH Editor authoring tool as reusable, granular and interoperable LOs.

\section{B. Structure}

LOs can be combined among them to build more complex ones forming a hierarchy. Regarding the aggregation levels (i.e. hierarchy levels) to which a LO belongs we can differentiate between coarse-grained and fine-grained or granular LOs [28]. Thereby, granular or fine-grained LOs are those that belong to the most granular or atomic aggregation level. The LOM [7] standard contemplates four aggregation levels to describe LO granularity.

The Virtual Excursion LO is defining conforming to its own learning object model. This model defines four levels of granularity based on the LOM standard with small differences according to the scheme of Fig. 2:

- The first level, which corresponds to the most granular or atomic level, includes raw media files like text, images, videos or flash objects and single elements like a website, a document or a $3 \mathrm{D}$ object. More sophisticated 


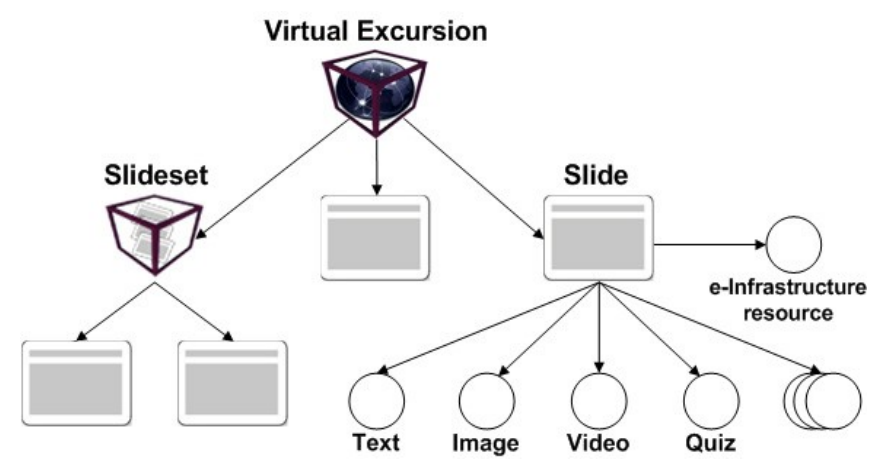

Fig. 2. Virtual Excursion aggregation levels

elements such as quizzes or e-Infrastructure resources belong to the first level too.

- The second level covers only one LO called "slide" that consists of a collection of level 1 LOs.

- The third level includes any LO that is built as a composition of level 2 LOs (i.e. "slides"). These LOs are identified under the name of "slidesets".

- Finally, the fourth level corresponds to the Virtual Excursion LO. A Virtual Excursion can contain level 3 LOs or directly a slide without using a slideset as a wrapper. Nevertheless, it cannot contain other Virtual Excursions. These are the main differences with respect to the LOM specification.

So, according to this structure, e-Infrastructure resources are fine-grained LOs. This means that they can be reused in multiple Virtual Excursions or slidesets through the slides. On the other hand, slides LOs can be used as components or building blocks of more complex LOs such as virtual experiments [29] or educational games [30].

Lastly, considering that LO metadata describes relevant characteristics of the LO to which it applies and can be used to facilitate its searching, retrieval and evaluation, Virtual Excursions are tagged with metadata based on LOM standard.

\section{VISH EDITOR}

ViSH Editor is a web-based e-Learning authoring tool that aims to create and edit Virtual Excursions. It is open source and the code is available at http://github.com/ging/vish editor. Today, it is available to end users through the ViSH platform.

\section{A. Interface and basic features}

ViSH Editor is based on the WYSIWYG (What You See Is What You Get) paradigm. It provides a usable and user friendly web interface designed with low-tech teaching contexts (where teachers are not computer specialists and also may have poor IT support) in mind. The user interface (UI) is mainly based on graphics but also have few textual elements (Fig. 3). ViSH Editor presents the Virtual Excursion as a slideshow, on which users can add new slides and move or remove the existing ones. Each slide is created from a template selected by the user. Inside a slide users can insert different resources and/or generate their own content. All of the inserted

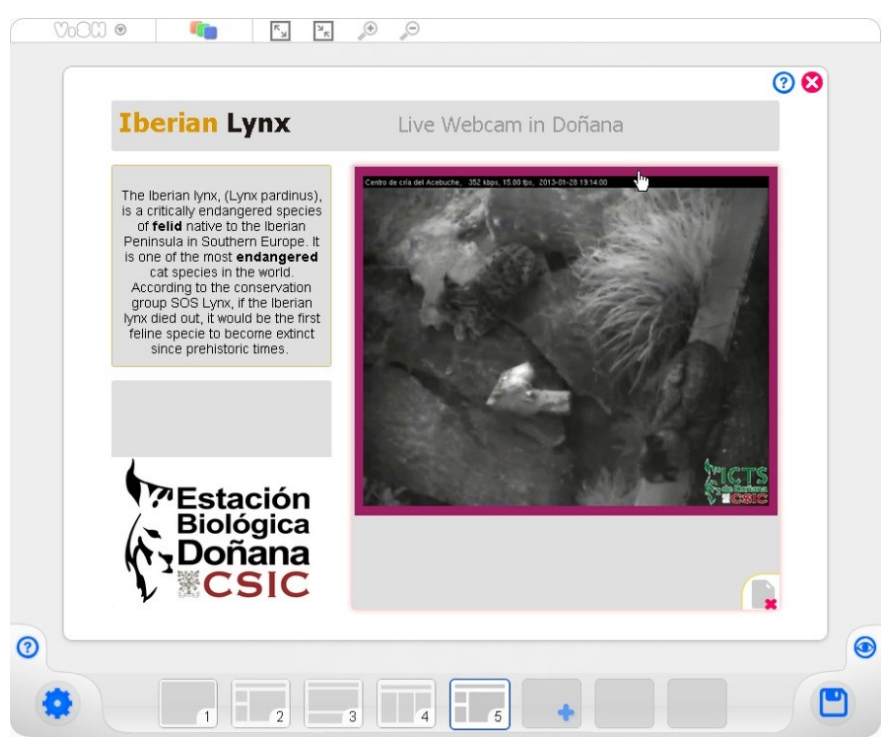

Fig. 3. User Interface of ViSH Editor

resources can be moved using the drag and drop technique. Furthermore, other actions can be performed over the distinct resources such as resize, zoom or hyperlink aggregation. Despite usability and user experience studies point out that ViSH Editor interface is very intuitive, a walkthrough is available for novice users to get started with the tool and learn new features. In addition, context-sensitive help is always available, providing help for the specific situation of the user. Users can preview the Virtual Excursion while they are creating it at any time. Also they can save the excursion as a draft to continue completing it later. In summary ViSH Editor assists teachers (or any other user) in the process of creating and publishing a Virtual Excursion.

\section{B. Resources and own content}

ViSH Editor allows users to insert many types of resources: images, videos, websites, PDF files, flash objects, etc. Any resource can be inserted via its URL. Also, ViSH Editor provides interfaces to search resources in the ViSH LOR as well as in external services like Flickr, YouTube or Vimeo.

Fig. 4 illustrates a search in the ViSH LOR. A catalogue of e-Infrastructures obtained from the ViSH LOR is also available. ViSH Editor facilitates the access and integration of these e-Infrastructures by managing the connections with live streamings and interacting with the web services and the particular interfaces of the e-Infrastructure resources. Moreover, users can upload their own resources to the ViSH. The slidesets and slides LOs of other Virtual Excursions can be also inserted. More types of resources are expected to be available to be integrated. For instance, the next coming version will have the functionality of insert and convert PowerPoint or PDF presentations in Virtual Excursions.

Besides adding resources, teachers can generate their own contents. They can insert paragraphs of text using a WYSIWYG rich text editor. Also, they can create their own quizzes to evaluate the acquired knowledge of the students. These quizzes can be inserted inside a slide as any other 


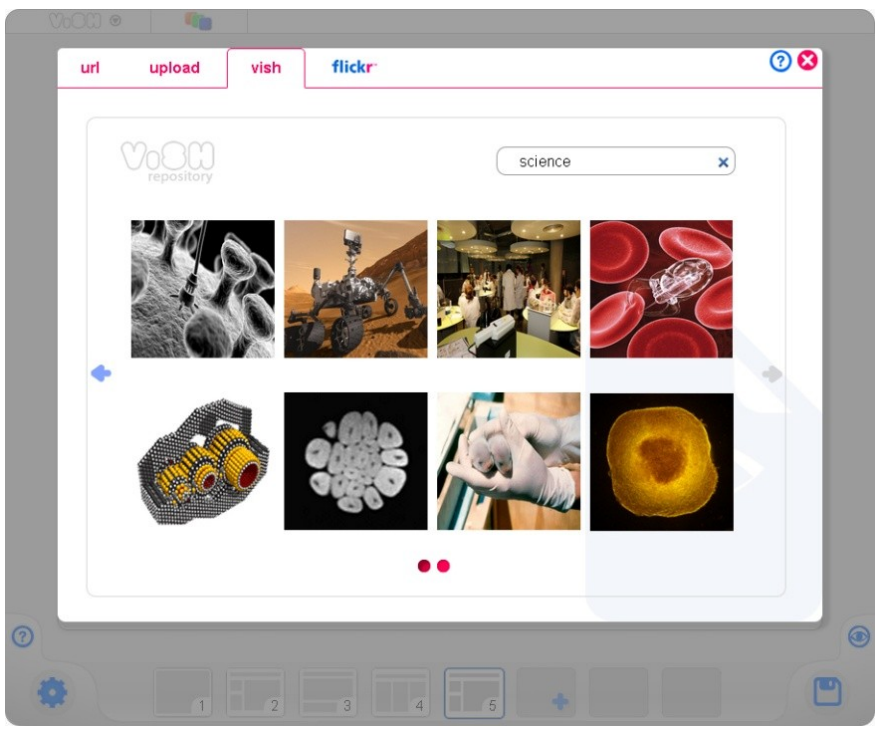

Fig. 4. Searching resources in ViSH Editor

content and hence they can be combined with any other resource. The questions can be true-false, multiple choice or open ended. Self-assessment is also possible. The results are stored and they can be accessed both in real time to be discussed in class or later to be analyzed in detail. The teacher can represent the data in many ways such as a bar graph or a pie chart.

\section{Metadata}

ViSH Editor also facilitates authors to fill the metadata of the Virtual Excursion LOs. They can indicate several metadata elements: Title, Description, Author, Language, Avatar (an image to identify the excursion visually), Tags (that act as keywords for the ViSH search engine) and others related to pedagogical qualities: Subject, Age Range, Educational Objectives and Acquired Competencies. There are other metadata elements that are inferred rather than specified by the author. For instance: metadata elements related to the structure or technical requirements, version, contributors, etc.

\section{Creating slidesets}

Besides creating Virtual Excursions, ViSH Editor can be used to generate other LOs called slidesets. These LOs are built using the slides LO as components and at the same time, they can be used to be part of an excursion as a conventional slide. Three slidesets can be created in the current version: flashcards [29], virtual tours and educational games [30].

To create a flashcard users select an image background (from ViSH LOR or Flickr, via URL or uploading it) and above it hot zones representing by arrows to link the slides. This way the slides will not have to be consumed in order but they will appear when touching or clicking on a hot zone.

Virtual tours are quite similar but instead of a static image background users can use an interactive map. They can search for any address and add locations representing by pins to which the slides will be linked.
Finally the same way the slides can be linked to hot zones in images or maps, they can be linked to actions or events in a game. A role-playing web game was developed where the teacher can link the slides to specific actions in the game, like "touch a blackboard" or "talk to some character". When the player performs any of these actions the slides appear. This way the game is customizable with the content from the slides.

\section{E. Interoperability}

The Virtual Excursions and their metadata are saved in the ViSH LOR in JSON format. However, in order to increase their interoperability, they can be downloaded as SCORM. So, they can be integrated in any Learning Management System (LMS) that supports this standard (e.g. Moodle or Blackboard). This way the students will have the excursions integrated with the rest of the resources that they use for their daily learning.

Moreover, all of the LOs generated by ViSH Editor provide an API (Application Programming Interface) which allows third party web applications to communicate with them and to use advanced functionalities (e.g. event notifications, advance slides, etc). Thereby they can be integrated in any website or web application. In the ViSH scenario this API has been used to integrate the Virtual Excursions with a videoconference tool called MashMe (http://mashme.tv). This integration allows teachers to share Virtual Excursions synchronously in real time with any other users.

\section{F. Technology and implementation}

ViSH Editor is a web application based on HTML5 [31], the new standard for the web. For this reason, any HTML5 compliant web browser can run the web tool and hence create Virtual Excursions. ViSH Editor has been developed as a JavaScript library and a set of HTML pages and CSS (Cascading Style Sheets) files. Despite it is a client-side application, some tasks (e.g. upload files) need a server backend. However, ViSH Editor is not tied to any specific backend technology, in fact we have developed two different implementations using Ruby on Rails and Node.js.

\section{G. Virtual Excursion examples}

Finally, to have a better understanding of how ViSH Editor can help teachers to create enhanced LOs through the use of e-Infrastructures resources, we present in this section some examples of Virtual Excursions.

The first example (illustrated in Fig. 5) is a Virtual Excursion to learn about physics in which students have the chance of accessing and controlling remotely three different instruments of a real physics laboratory: a pendulum, a pulley and a spring. First off to create this excursion, the teacher has written some physics exercises which are formulated at the beginning. Then, he/she has searched and included the e-Infrastructure resources, which were previously uploaded to ViSH by BIFI. Lastly, the teacher has created some quizzes to collect their students' answers. This way the students can compare their theoretical calculations with the measurements of the real systems before they answer the quizzes.

Many other Virtual Excursions have been created in the ViSH platform by teachers and scientific organizations. 

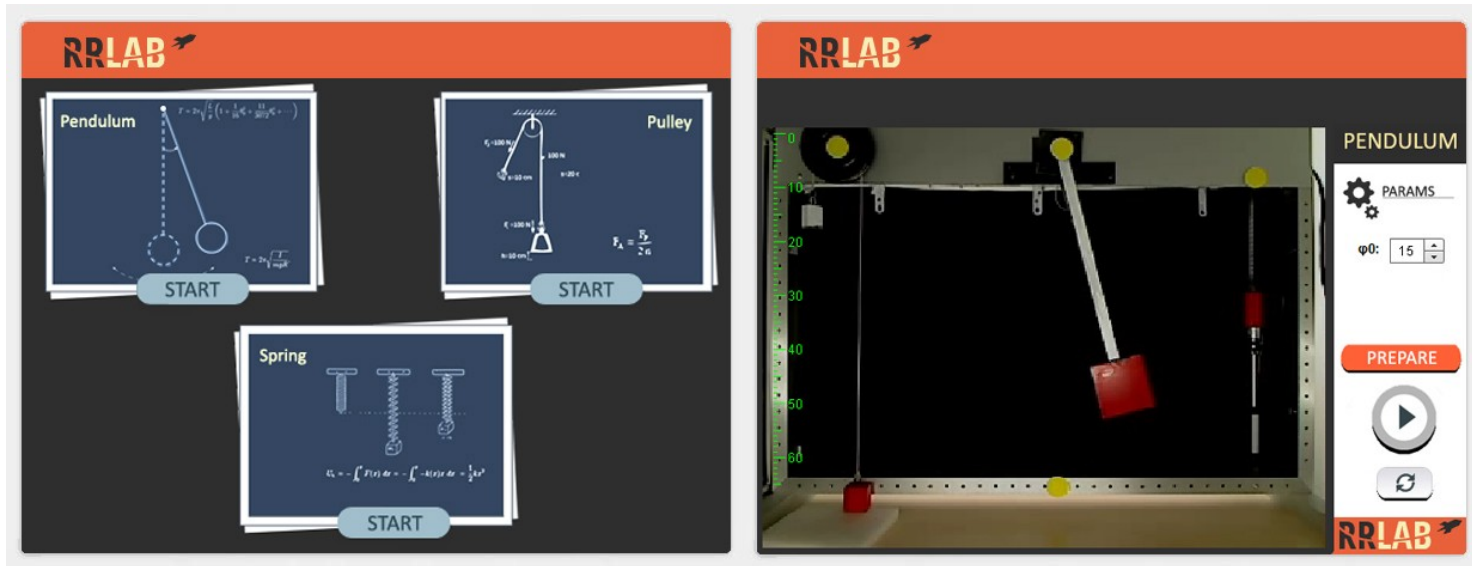

Fig. 5. Virtual Excursion of a Remote Physics Laboratory

For instance, a virtual visit to the Doñana Biological Reserve where students can observe a huge variety of animal species through live webcams.

Another interesting example is one where students can observe in real time the video output of different microscopes provided by the Nanoscience Centre of UCAM as well as receive explanations of the researcher who will be operating the microscope.

\section{CONCLUSIONS AND FUTURE WORK}

In this paper we have presented ViSH Editor, an innovative web-based e-Learning authoring tool to create enhanced LOs called Virtual Excursions using e-Infrastructure resources. ViSH Editor creates Virtual Excursions as reusable and granular LOs. It also provides several mechanisms to integrate them into websites, third party services and LMSs. Lastly, we have exposed some examples of how to enrich learning using Virtual Excursions.

From our experience with ViSH Editor, where we had to develop several tailored solutions to facilitate access to selected e-Infrastructures, we believe that providing a standardized way to access e-Infrastructures resources is one of the main challenges to promote the use of e-Infrastructures in education.

On the other hand, an authoring tool should be adapted to the needs of their end users to be successful. So, if the end user is a teacher, who does not have to be a computer specialist, the authoring tool must provide a simple, intuitive and user friendly interface including context-sensitive help and optional walkthroughs.

Authoring tools can play an important role in modern e-Learning platforms creating and maintaining didactic contents. In collaborative environments, the reusability of these contents is the cornerstone. Thereby these contents should be created following a learning object model that defines appropriately the granularity levels, the structure and the metadata. Moreover, several efforts should be undertaken to achieve interoperability, especially with LMSs by using e-Learning standards.
The Virtual Excursions created via ViSH Editor are a great example of how technology can enhance learning, but there are some future works that can be performed to bring new contributions in order to keep improving.

First off, based on our experiences, we plan to define a standard for e-Infrastructure access and develop and test it in real cases. Also, we want to define a model to integrate the existing learning objects into games, building a bridge between game developers and teachers. Finally we will carry out an evaluation of the use of the Virtual Excursions in the classroom to measure their educational impact.

\section{ACKNOWLEDGMENT}

We wish to acknowledge our gratitude and appreciation to all the GLOBAL excursion project partners, and each one of the project team members, for their contribution during the development of various ideas and concepts presented in this paper. We also would like to thank the SAAN project (TIN2010-19138) for funding this work.

\section{REFERENCES}

[1] E. Commission, "Supporting the Teaching Professions for Better Learning Outcomes," 2012.

[2] C. Stewart, "What is Cyberinfrastructure?" [Online]. Available: http://hdl.handle.net/2022/13987.

[3] S. Ainsworth, M. Honey, W. L. Johnson, K. Koedinger, B. Muramatsu, R. Pea, M. Recker, and S. Weimar, "Cyberinfrastructure for Education and Learning for the Future: A vision and research agenda," 2005.

[4] D. Mcarthur, "National Science Digital Library: Shaping Education's Cyberinfrastructure," IEEE Computer, 2008.

[5] S. Itamar, B. David, D. Israel, and K. Arik, "Do eLearning Technologies Improve the Higher Education Teaching and Learning Experience?," in Proceedings of the Fifth International Conference on eLearning for Knowledge-Based Society, 2008.

[6] A. R. Hurson, S. Sedigh, L. Miller, and B. Shirazi, "Enriching STEM education through personalization and teaching collaboration," in Proceddings of the 7th IEEE International Conference on Pervasive Computing and Communications Workshops (PERCOM Workshops), 2011, pp. 543-549.

[7] IEEE LTSC, "Draft Standard for Learning Object Metadata," 2002 
[8] K. Verbert and E. Duval, "ALOCOM: a generic content model for learning objects," International Journal on Digital Libraries, vol. 9, no. 1, pp. 41-63, 2008.

[9] D. A. Wiley, "Learning Object Design and Sequencing Theory," Brigham Young University, 2000.

[10] P. Mohan and C. Brooks, "Learning Objects on the Semantic Web," in Proceedings of the 3rd IEEE International Conference on Advanced Technologies, 2003, pp. 195-199.

[11] E. Duval, K. U. Leuven, and W. Hodgins, "A LOM Research Agenda," in Proceedings of the 12th International World Wide Web Conference (2003), 2003, pp. 1-9.

[12] A. Littlejohn, I. Falconer, and L. Mcgill, "Characterising effective eLearning resources," Computers \& Education, vol. 50, no. 3, pp. 757-771, Apr. 2008.

[13] S. Schluep, Modularization and structured markup for Web-based Learning Content in an Academic Environment. 2005.

[14] “Advanced Distributed Learning (ADL), SCORM 2004 4th Edition,' 2004. [Online]. Available: http://www.adlnet.gov/capabilities/scorm/scorm-2004-4th.

[15] M. Haghshenas, M. Khademi, and H. Kabir, "E-learning and Authoring Tools: At a Glance," International Journal of Research \& Reviews in Applied Sciences, vol. 10, no. 2, pp. 259-263, 2012.

[16] A. Fernández-Pampillón Cesteros, E. Domínguez, J. M. Lahoz Bengoechea, D. Romero López, I. de Armas Ranero, S. Palmaz, and J. Arús Hita, "A Strategy for the Inductive Generation of Learning Objects in low-Tech Contexts," in Proceedings of the 10th European Conference on E-Learning (ECEL 2011), 2011.

[17] "Distributed European Infrastructure for Supercomputing Applications (DEISA).” [Online]. Available: http://www.deisa.eu.

[18] "European Grid Infrastructure (EGI)." [Online]. Available: http://www.egi.eu.

[19] “Open Science Grid (OSG).” [Online]. Available: http://www.opensciencegrid.org.

[20] "Extreme Science and Engineering Discovery Environment (XSEDE)," [Online]. Available: http://www.xsede.org.

[21] W. Gentzsch, "Building sustainable e-Infrastructures for Research and Education. DEISA Project.” 2011.

[22] T. Hey and A. E. Trefethen, "Cyberinfrastructure for e-Science," 2005
[23] E. Commission, "Communication from the Commission to the European Parliament, the Council, the European Economic and Social Committee and the Committee of the Regions: Rethinking Education: Investing in skills for better socio-economic outcomes," 2012.

[24] A. Gordillo, E. Barra, and J. Quemada, "Enhancing K-12 science education through a multi-device web tool to facilitate content integration and e-Infrastructure access," in Proceedings of the 7th International Technology, Education and Development Conference (INTED 2013), 2013.

[25] T. Holocher-ertl, B. Kieslinger, and C. M. Fabian, "Linking schools with science: How innovative tools can increase the effectiveness of science teaching in the classroom," 2012.

[26] A. Tapiador, D. Carrera, and J. Salvachúa, "Social Stream, a social network framework," in Proceedings of the 1th International Conference on Future Generation Communication Technology (FGCT 2012), 2012.

[27] B. Kieslinger, T. Holocher, C. M. Fabian, D. Gallego, S. Aguirre, E. Barra, and G. Mihai, "Virtual Excursions: a New Way to Explore Science in Class," in Proceddings of the 2th International Conference on New Perspectives in Science Education (NPSE 2013), 2013.

[28] S. Fadzilah, N. Yusof, and S. Zaiton, "Creating Granular Learning Object Towards Reusability of Learning Object In E-learning Context," in Proceedings of the 2011 International Conference on Electrical Engineering and Informatics (2011), 2011.

[29] E. Barra, D. Gallego, S. Aguirre, and J. Quemada, "Facilitating the creation of K-12 interactive learning objects using a multi device web tool," in Proceedings of the 2012 Frontiers in Education Conference (FIE 2012), 2012.

[30] E. Barra, D. Gallego, S. Aguirre, and J. Quemada, "A web tool to create educational content with gaming visualization," in Proceedings of the 2012 Frontiers in Education Conference (FIE 2012), 2012.

[31] W3C, "HTML5 specification." [Online]. Available: http://www.w3.org/html/wg/drafts/html/master/Overview.html. 